\title{
L. Bădescu
}

Nagoya Math. J.

Vol. 86 (1982), 155-171

\section{ON AMPLE DIVISORS}

\author{
LUCIAN BǍDESCU
}

\section{Introduction}

In this paper we are dealing with the following problem: determine all normal (or smooth) projective varieties $X$ over an algebraically closed field $k$ supporting a given variety $Y$ as an ample Cartier divisor. In $\S 1$ we assume $Y=P^{n-1}$ with $n \geqslant 3$ and show that such a normal variety $X$ is isomorphic to the projective cone over $v_{s}(Y)$, where $s>0$ is the integer determined by the equality

$$
O_{X}(Y) \otimes O_{Y}=O(s) \quad \text { and } \quad v_{s}: P^{n-1} \hookrightarrow P^{N-1}\left(N=\left(\begin{array}{c}
n+s-1 \\
n-1
\end{array}\right)\right)
$$

is the $s^{\text {th }}$ Veronese embedding of $P^{n-1}$. A similar result is valid for $Y=$ $P^{s} \times P^{t}$ with $s, t \geqslant 2$. In the second section we prove the following generalization of a result of Sommese ([13]). If $Y=H(d)$ is a hypersurface of prime degree $d$ in $P^{n+1}$ such that either $n \geqslant 3$, or else char $(k)=0$ and $H(d)$ is a generic surface in $P^{3}$ with $d \geqslant 5$, then $Y$ can be contained in a smooth projective variety $X$ as an ample divisor only in one of the following two cases: i) $X$ is $P^{n+1}$ and the inclusion $Y \subset X$ is just the inclusion $H(d) \subset P^{n+1}$, or ii) $X$ is a smooth hypersurface of degree $d$ in $P^{n+1}$ and $Y$ is the intersection of $X$ with a hyperplane. In the last section we determine all smooth projective threefolds $X$ with $P^{2}$ (resp. $P^{1} \times P^{1}$ ) as an ample divisor. Note that if char $(k)=0$ the proofs are not so complicated (in the case of $Y=P^{2}$ the result being (well known and) contained in $\S 1$ ) because one applies the result of [2]. However, by the method of lifting to characteristic zero we show that in our situation we can apply [2] in positive characteristic as well.

The proofs of these results require Lefschetz type theorems in Grothendieck's form ([7], [8], [2]). Throughout this paper $k$ will be an algebraically closed field of arbitrary characteristic and the notations and terminology will be standard, unless otherwise specified.

Received April 25, 1980. 


\section{$\S 1$. Normal projective varieties containing $P^{n-1}(n \geqslant 3)$ or $P^{s} \times P^{t}$ $(s, t \geqslant 2)$ as an ample Cartier divisor}

Let $Y$ be an arbitrary connected smooth projective variety over $k$ and choose a projectively normal embedding $i: Y \smile P^{m}$ of $Y$ (by a theorem of Serre such an embedding always exists). Denote by $C(Y, i)$ the projective cone in $P^{m+1}$ over $i(Y)$. Then $C(Y, i)$ is a normal projective variety containing $i(Y)$ as an ample Cartier divisor.

ExAmples. 1) Take $Y=P^{n-1}$ with $n \geqslant 2$ and for every $s>0$ consider the $s^{\text {th }}$ Veronese embedding

$$
v_{s}: P^{n-1} \longleftrightarrow P^{N-1} \text { with } \quad N=\left(\begin{array}{c}
n+s-1 \\
n-1
\end{array}\right) .
$$

Then $v_{s}$ is projectively normal and hence $P^{n-1}$ is an ample Cartier divisor in the normal variety $X_{s}^{n}=C\left(P^{n-1}, v_{s}\right)$ such that the normal sheaf $N_{P^{n-1, X} X_{s}^{n}}$ is $O(s)=O_{P^{n-1}}(s)$. Moreover, $X_{1}^{n}=P^{n}$.

2) Take $Y=P^{s} \times P^{t}$ with $s \geqslant 2$ and $t \geqslant 2$ and for every $a>0, b>0$ consider the Segre-Veronese embedding

$$
i_{a, b}: P^{s} \times P^{t} \longrightarrow P^{N-1} \quad \text { with } \quad N=\left(\begin{array}{c}
s+a \\
s
\end{array}\right)\left(\begin{array}{c}
t+b \\
b
\end{array}\right) .
$$

Then $i_{a, b}$ is projectively normal and hence $Y$ is an ample Cartier divisor on the cone $C\left(P^{s} \times P^{t}, i_{a, b}\right)=X_{a, b}^{s, t}$ such that the respective normal sheaf if $O(a, b)=p_{1}^{*}\left(O_{P^{s}}(a)\right) \otimes p_{2}^{*}\left(O_{P^{t}}(b)\right), p_{1}$ and $p_{2}$ being the canonical projections of $P^{s} \times P^{t}$.

Theorem 1. Assume that $n \geqslant 4$ and that $Y=P^{n-1}$ is an ample Cartier divisor on the normal projective variety $X$. Then if the normal sheaf $N_{Y, X}$ is isomorphic to $O(s)$ (necessarily $s>0$ ), $X$ is isomorphic to $X_{s}^{n}$ and $Y$ is contained in $X$ as in example 1 above. If $n=3$ the same conclusion holds provided that $\operatorname{char}(k)=0$. In particular, $X$ is smooth if and only if $s=1$, i.e. $X=P^{n}$.

Proof. Let $\operatorname{Sing}(X)$ be the singular locus of $X$ and set $U=X-$ Sing $(X)$. Since $Y$ is a smooth Cartier divisor on $X, Y \subset U$, and since $Y$ is ample, $\operatorname{dim}(\operatorname{Sing}(X)) \leqslant 0$, i.e. Sing $(X)$ consists of at most a finite set of closed points $\left\{x_{1}, \cdots, x_{h}\right\}$.

By [7], expose $X$, Example 2.2 the pair $(X, Y)$ satisfies the effective Lefschetz condition, Leff $(X, Y)$. Since this condition is local along $Y$ we 
have also Leff $(U, Y)$. If $n \geqslant 4$ we have $H^{i}\left(O_{X}(-m Y) / O_{X}(-(m+1) Y)\right)=$ $H^{i}(O(-m s))=0$ for $i=1,2$ and for every $m>0$. Hence by [7], exposé $\mathrm{XI}$, théorème 3.12 the natural map of restriction $\alpha$ : $\operatorname{Pic}(U) \rightarrow \operatorname{Pic}(Y) \cong Z$ is an isomorphism. If instead $n=3$ and $\operatorname{char}(k)=0$ we have

$$
H^{1}\left(O_{X}(-m Y) / O_{X}(-(m+1) Y)\right)=H^{1}(O(-m s))=0
$$

for every $m>0$, and then apply the theorem of [2] (in a slightly modified form) to deduce that $\alpha$ is injective and Coker $(\alpha)$ is torsion-free. Since $\operatorname{Pic}(U) \neq 0\left(O_{X}(Y) / U \not O_{U}\right)$ and Pic $(Y) \cong Z$ this yields that $\alpha$ is also an isomorphism.

Therefore in both cases there is an invertible $O_{U}$-module $L$ such that $L \otimes O_{Y}=O(1)$. For every $m \in Z$ put $F^{(m)}=j_{*}\left(L^{\otimes m}\right)$, where $j: U \smile X$ is the canonical open immersion. The following statements hold:

a) $F^{(m)}$ is a coherent $O_{X}$-module and $\operatorname{depth}_{o_{x_{i}}}\left(\left(F^{(m)}\right)_{x_{i}}\right) \geqslant 2$ for every $m \in \boldsymbol{Z}$.

Indeed, the coherence of $F^{(m)}$ comes from [7], exposé VIII, Corollary VIII-II-3. On the other hand, the canonical map $F^{(m)} \rightarrow j_{*} j^{*}\left(F^{(m)}\right)$ is (by the very definition of $F^{(m)}$ ) an isomorphism, and the second affirmation follows from the exact sequence

$$
0 \longrightarrow \bigoplus_{i=1}^{h} H_{x_{i}}^{0}\left(\left(F^{(m)}\right)_{x_{i}}\right) \longrightarrow F^{(m)} \longrightarrow j_{*} j^{*}\left(F^{(m)}\right) \longrightarrow \bigoplus_{i=1}^{h} H_{x_{i}}^{1}\left(\left(F^{(m)}\right)_{x_{i}}\right) \longrightarrow 0 .
$$

b) $F^{(m s)} \cong O_{X}(m Y)$ for every $m \in Z$.

Indeed, $L^{\otimes m s} \cong O_{X}(m Y) / U$ because $O_{X}(m Y) \otimes O_{Y}=O(m s)$ and the map $\alpha$ is injective. Applying $j_{*}$ to this isomorphism and taking into account that depth $\left(O_{x_{i}}\right) \geqslant 2\left(O_{x_{i}}\right.$ is normal of dimension $\left.\geqslant 2\right)$ we get the conclusion.

c) $H^{1}\left(F^{(m)}\right)=0$ for every $m \ll 0$.

First choose $t$ big enough so that $O_{X}(t Y)$ is very ample and consider the embedding $i: X \smile P=P\left(\Gamma\left(X, O_{X}(t Y)\right)\right.$ such that $i^{*} O_{P}(1) \cong O_{X}(t Y)$.

Claim. For every coherent $O_{X}$-module $G$ such that $\operatorname{depth}_{o_{x}}\left(G_{x}\right) \geqslant 2$ for every closed point $x \in X, H^{1}\left(G \otimes O_{X}(q Y)\right)=0$ for every $q \ll 0$.

Proof of the claim. Set $G^{\prime}=i_{*}(G)$. For every closed point $y \in P-$ $i(X)$ we have clearly $H_{y}^{1}\left(G_{y}^{\prime}\right)=0$. If $y \in i(X)$ is a closed point, by [5], Corollary 5.6 we have $H_{y}^{1}\left(G_{y}^{\prime}\right)=H_{y}^{1}\left(G_{y}\right)$, and recalling the hypothesis the last group is zero. Thus we may apply [7], exposé XII, Corollary 1.3 and deduce that $H^{1}\left(X, G \otimes O_{X}\left(q^{\prime} t Y\right)\right)=H^{1}\left(P, G^{\prime} \otimes O_{P}\left(q^{\prime}\right)\right)=0$ for every $q^{\prime} \ll 0$. Also, denoting by $G_{r}=G \otimes O_{X}(r Y), r=0,1, \cdots, t-1\left(G_{0}=G\right)$, then 
$H^{1}\left(X, G_{r} \otimes O_{X}\left(q^{\prime} t Y\right)\right)=0$ for $q^{\prime} \ll 0$ (because for every closed point $x \in X$ depth $\left.\left(\left(G_{r}\right)_{x}\right) \geqslant 2\right)$. Now let $q$ be arbitrary and divide $q=q^{\prime} t+r$, with $0 \leqslant r \leqslant t-1$. The equality $G \otimes O_{X}(q Y)=G_{r} \otimes O_{X}\left(q^{\prime} t Y\right)$ and the above discussion proves the claim.

Now in order to prove c) write $m=q s+r$, with $0 \leqslant r \leqslant s-1$. Since $O_{X}(Y)$ is invertible on $\left.X, \mathrm{~b}\right)$ and projection formula yield

$$
\begin{aligned}
F^{(m)} & =j_{*}\left(L^{\otimes r} \otimes L^{\otimes q s}\right)=j_{*}\left(L^{\otimes r} \otimes j^{*}\left(O_{X}(q Y)\right)\right) \cong j_{*}\left(L^{r}\right) \otimes O_{X}(q Y) \\
& =F^{(r)} \otimes O_{X}(q Y) .
\end{aligned}
$$

The statement of c) follows applying the claim to $G=F^{(r)}, r=$ $0,1, \cdots, s-1$ and taking into account a).

d) Let $\sigma \in \Gamma\left(X, F^{(s)}\right) \cong \Gamma\left(X, O_{X}(Y)\right)$ be such that $\operatorname{div}_{X}(\sigma)=Y$. Then for every $m \in Z$ there is the exact sequence on $X$

$$
0 \longrightarrow F^{(m-s)} \stackrel{\sigma}{\longrightarrow} F^{(m)} \longrightarrow O(m) \longrightarrow 0,
$$

where the first map is multiplication by $\sigma$.

Indeed, the exact sequence

$$
0 \longrightarrow O_{X}(-Y) \stackrel{\sigma}{\longrightarrow} O_{X} \longrightarrow O_{Y} \longrightarrow 0
$$

tensorized by $F^{(m)}$ yields the exact sequence

$$
F^{(m)} \otimes O_{X}(-Y) \cong F^{(m-s)} \stackrel{\sigma}{\longrightarrow} F^{(m)} \longrightarrow O(m) \longrightarrow 0 .
$$

Since $F^{(m)}$ is invertible on $U$ the map $\sigma / U$ is injective, and since $\sigma\left(x_{i}\right)$ $\neq 0$ for every $i=1, \cdots, h, \sigma$ is injective everywhere.

Now (1) yields the exact sequence of cohomology $(m \in Z)$

$$
\begin{aligned}
0 \longrightarrow \Gamma\left(X, F^{(m-s)}\right) \stackrel{\sigma}{\longrightarrow} \Gamma\left(X, F^{(m)}\right) \longrightarrow \Gamma(Y, O(m)) \\
\longrightarrow H^{1}\left(X, F^{(m-s)}\right) \stackrel{\psi_{m}}{\longrightarrow} H^{1}\left(X, F^{(m)}\right) \longrightarrow H^{1}(Y, O(m))=0 .
\end{aligned}
$$

Thus for every $m \in Z$ the map $\psi_{m}$ is surjective. Thus from c) and induction on $m$ it follows that $H^{1}\left(X, F^{(m)}\right)=0$ for every $m \in Z$. Thus for every $m$ one gets the exact sequence

$$
0 \longrightarrow \Gamma\left(X, F^{(m-s)}\right) \stackrel{\sigma}{\longrightarrow} \Gamma\left(X, F^{(m)}\right) \longrightarrow \Gamma(Y, O(m)) \longrightarrow 0 .
$$

Set $S=\oplus_{m=0}^{\infty} \Gamma\left(X, F^{(m)}\right)=\bigoplus_{m=0}^{\infty} \Gamma\left(U, L^{\otimes m}\right)$. Then $S$ is a graded $k$ algebra, $\sigma \in S_{s}$ and (2) yields the isomorphism of graded $k$-algebras 
$S / \sigma S \cong \bigoplus_{m=0}^{\infty} \Gamma(Y, O(m)) \cong k\left[T_{1}, \cdots, T_{n}\right]$ (polynomial ring in $n$ variables)

Set $S^{\prime}=S^{(s)}$, where $S_{t}^{\prime}=S_{s t}$ for every $t \in Z$. Then $\sigma \in S_{1}^{\prime}$ and

$$
S^{\prime} / \sigma S^{\prime}=k\left[T_{1}, \cdots, T_{n}\right]^{(s)}
$$

Choose $t_{i} \in S_{1}$ such that $t_{i} \bmod \sigma S=T_{i}$ and set $\sigma_{i_{1}, \cdots, i_{n}}=t_{1}^{i_{1}} \cdots t_{n}^{i_{n}} \in S_{s}$ $=S_{1}^{\prime}$, where $i_{1}+\cdots+i_{n}=s$ and $i_{m} \geqslant 0$. Then $\sigma_{i_{1}, \cdots, i_{n}}$ satisfy the well known Veronese equations

$$
\sigma_{i_{1}, \cdots, i_{n}} \cdot \sigma_{j_{1}, \cdots, j_{n}}-\sigma_{e_{1}, \cdots, e_{n}} \cdot \sigma_{f_{1}, \cdots, f_{n}}=0
$$

where $i_{m}+j_{m}=e_{m}+f_{m}, m=1, \cdots, n$.

Furthermore the images of $\left\{\sigma_{i_{1}, \cdots, i_{n}}\right\}$ in $S^{\prime} / \sigma S^{\prime}$ generate the graded $k$ algebra $S^{\prime} / \sigma S^{\prime}$, and since $\sigma \in S_{1}^{\prime}$, it follows that $\sigma$ and $\left\{\sigma_{i_{1}, \cdots, i_{n}}\right\}$ generate $S^{\prime}$ as a graded $k$-algebra.

In particular, $S^{\prime}=\bigoplus_{m=0}^{\infty} \Gamma\left(X, O_{X}(m Y)\right)$ is generated by its part of degree one. Since $Y$ is ample on $X, O_{X}(Y)$ results then very ample. Thus the canonical map $\varphi_{Y}: X \rightarrow P\left(\Gamma\left(X, O_{X}(Y)\right)\right.$ ) (such that $\varphi_{Y}^{*}(O(1)) \cong O_{X}(Y)$ ) is a closed immersion. If in (2) we take $m=s$ we get $\operatorname{dim} \Gamma\left(X, O_{X}(Y)\right)=$ $\operatorname{dim} \Gamma\left(X, O_{X}\right)+\operatorname{dim} \Gamma(Y, O(s))=N+1$, where

$$
N=\left(\begin{array}{c}
n+s-1 \\
n-1
\end{array}\right)
$$

Thus $\varphi_{Y}(X) \subset P^{N}$ and $\varphi_{Y}$ restricted to $Y$ is precisely the Veronese embedding $v_{s}$. In particular, $Y$ is the intersection of $X$ with the hyperplane $P^{N-1}$. It remains to be proved that $\varphi_{Y}(X)$ is isomorphic to the cone $X_{s}^{n}$.

Set $S^{\prime \prime}=k\left[T_{1}, \cdots, T_{n}\right]^{(s)}$, grade the polynomial $k$-algebra $S^{\prime \prime}[T]$ so that if $a \in S^{\prime \prime}$ is an arbitrary homogeneous element then $\operatorname{deg}\left(a T^{m}\right)=$ $\operatorname{deg}(a)+m$, and define the homomorphism of graded $k$-algebras $\psi: S^{\prime \prime}[T]$ $\rightarrow S^{\prime}$ by $\psi(T)=\sigma$ and $\psi\left(T_{1}^{i_{1}} \cdots T_{n}^{i_{n}}\right)=\sigma_{i_{1}, \cdots, i_{n}}$, where $i_{m} \geqslant 0$ and $i_{1}+\cdots$ $+i_{n}=s$. The equations (3) ensure us that this definition is correct. Since $\sigma_{i_{1}, \cdots, i_{n}}$ and $\sigma$ generate $S^{\prime}$ as a $k$-algebra, $\psi$ is surjective. Also, the dimension of $S^{\prime \prime}[T]$ and $S^{\prime}$ are the same (namely $n+1$ ) and these graded algebras are integral domains. Therefore $\psi$ is an isomorphism, which proves that $\varphi_{Y}(X) \cong X_{s}^{n}$.

Q.E.D.

Exactly in the same way one can prove the following theorem.

Theorem 2. Assume that $Y=P^{s} \times P^{t}$ (with $s \geqslant 2$ and $t \geqslant 2$ ) is an 
ample divisor on the normal projective variety $X$. Then if the normal sheaf $N_{Y, X}$ is isomorphic to $O(a, b)$ (necessarily $a>0$ and $b>0$ ), $X$ is isomorphic to the cone $X_{a, b}^{s, t}$ (from Example 2 above). In particular, $P^{s} \times$ $P^{t}$ cannot be contained in a smooth projective variety as an ample divisor.

Remark. The assumption about the normality of $X$ in Theorem 1 or Theorem 2 cannot be dropped. Indeed, consider the Veronese embedding $v_{2}: P^{2} \hookrightarrow P^{5}$ and take the generic projection $Y^{\prime}$ of $v_{2}\left(P^{2}\right)$ into $P^{4}$, i.e. the Veronese surface in $P^{4}$. Then $Y^{\prime}$ is isomorphic to $P^{2}, Y^{\prime}$ is an ample Cartier divisor on the cone $C\left(Y^{\prime}\right) \subset P^{5}$ over $Y^{\prime}$, but since $Y^{\prime}$ is the projection of $v_{2}\left(P^{2}\right)$ into $P^{4}$, the vertex of $C\left(Y^{\prime}\right)$ is not a normal point. Thus $C\left(Y^{\prime}\right)$ cannot be isomorphic to any $X_{s}^{3}$.

Corollary 1. i) Assume that $Y=P^{n-1}$ is an effective Cartier divisor on the normal complete variety $X$ such that $N_{Y, X}=O(s)$ with $s>0$, and assume moreover that either $n \geqslant 4$, or else $n=3$ and char $(k)=0$. Then there is a birational morphism $f: X \rightarrow X_{s}^{n}$ such that $f$ is an isomorphism in $a$ neighbourhood of $Y$ and $f(Y)=v_{s}\left(P^{n-1}\right)$.

ii) Assume that $Y=P^{s} \times P^{t}(s \geqslant 2, t \geqslant 2)$ is an effective Cartier divisor on the normal complete variety $X$ such that $N_{Y, X}=O(a, b)$ with $a>0$ and $b>0$. Then there is a birational morphism $f: X \rightarrow X_{a, b}^{s, t}$ such that $f$ is an isomorphism in a neighbourhood of $Y$ and $f(Y)=i_{a, b}\left(P^{s} \times P^{t}\right)$.

Proof. Let us prove for example i). By [8], chapter III, Theorem 4.2 there is a birational morphism $f: X \rightarrow X^{\prime}$ such that $f$ is an isomorphism in a neighbourhood of $Y$ and $Y^{\prime}=f(Y)$ is an ample Cartier divisor on $X^{\prime}$. Since $X$ is normal, we may assume that $X^{\prime}$ is also normal. Then by Theorem $1 X^{\prime} \cong X_{s}^{n}$ such that $Y^{\prime}$ corresponds to $v_{s}\left(P^{n-1}\right)$. Q.E.D.

Corollary 2. Assume that $Y$ is as in Corollary $1 \mathrm{i})$ or ii), and let $Y \subset X_{i}(i=1,2)$ two closed immersions such that $X_{1}$ and $X_{2}$ are smooth varieties of dimension equal to $\operatorname{dim}(Y)+1$ and $N_{Y, X_{1}} \cong N_{Y, X_{2}}$ is ample. Then there is a birational map $u: X_{1} \rightarrow X_{2}$ which is an isomorphism on an open neighbourhood of $Y$ in $X$ and induces identity on $Y$.

\section{§2. A generalization of a result of Sommese}

First we need the following extension to arbitrary characteristic of a result of Kobayashi-Ochiai (see [11]). For the intersection theory of line bundles needed in this section we refer to [10]. 
TheOREM 3 (Kobayashi-Ochiai). Let $V$ be a complete Cohen-Macaulay algebraic scheme of pure dimension $t>0$ over $k$ and $L$ an ample invertible $O_{V}$-module such that $\left(L^{\cdot t}\right)_{V}=1$ and $\operatorname{dim} \Gamma(V, L) \geqslant t+1$. Then $\operatorname{dim} \Gamma(V, L)$ $=t+1$ and the canonical map $\varphi_{L}: V \rightarrow P(\Gamma(V, L)) \cong P^{t}$ is a biregular isomorphism.

Proof. First we prove that $V$ is integral. Let $V_{1}, \cdots, V_{n}$ be the irreducible components of $V$ naturally regarded as closed subschemes of $V$ (see [10], p. 298). Then by loc. cit. Proposition 5 and Corollary 1 one has

$$
\left(L^{\cdot t}\right)_{V}=\left(L^{\cdot t}\right)_{V_{1}}+\cdots+\left(L_{n}^{\cdot t}\right)_{V_{n}}, \quad \text { where } L_{i}=L \otimes O_{V_{i}} .
$$

Since every $V_{i}$ has dimension $t$ and $L_{i}$ is ample on $V_{i},\left(L_{i}^{\cdot t}\right)_{V_{i}}>0$ for every $i=1, \cdots, n$. Thus if $V$ were reducible the above equality would imply $\left(L^{\cdot t}\right)_{V} \geqslant 2$, a contradiction.

Thus $V$ is irreducible. By loc. cit. Proposition 5 and Corollary 2 (p. 298) one has

$$
\left(L^{t}\right)_{V}=\text { length }\left(O_{V, \xi}\right) \cdot\left(M^{\cdot t}\right)_{V_{\text {red }}},
$$

where $M=L \otimes O_{V_{\text {red }}}$ and $\xi$ is the generic point of $V$. Thus length $\left(O_{V, \xi}\right)$ $=1$, i.e. $V$ is generically reduced. Now since $V$ is Cohen-Macaulay and generically reduced, [1], chap. VII, Proposition 2.2 shows that $V$ is reduced everywhere. Thus $V$ is integral.

Let now $s_{1}, \cdots, s_{t+1}$ be $t+1$ linearly independent section (over $k$ ) from $\Gamma(V, L)$ and $D_{i}=\operatorname{div}_{V}\left(s_{i}\right)$. Define the sequence of closed subsets of $V$

$$
V=V_{t} \supseteq V_{t-1} \supseteq \cdots \supseteq V_{0} \supseteq V_{-1}
$$

by $V_{t-i}=D_{1} \cap \cdots \cap D_{t}$ for $i=1, \cdots, t+1$. $\quad V_{t-i}$ can be naturally endowed with a structure of closed subscheme of $V, i=1, \cdots, t+1$. Then one can easily prove as before that each $V_{t-i}$ is an integral CohenMacaulay scheme of dimension $t-i$ and that there is a natural exact sequence

$$
0 \longrightarrow\left(s_{1}, \cdots, s_{i}\right) \longrightarrow \Gamma(V, L) \longrightarrow \Gamma\left(V_{t-i}, L \otimes O_{v_{t-i}}\right),
$$

where $\left(s_{1}, \cdots, s_{i}\right)$ is the subspace of $\Gamma(V, L)$ generated by $s_{1}, \cdots, s_{i}$ (see [11] for details). From this point one gets the conclusion exactly as in [11].

Q.E.D.

Theorem 4. Let $Y=H(d)$ be a hypersurface of $P^{n+1}$ (i.e. a complete intersection of codimension one in $P^{n+1}$, not necessarily smooth) of degree 
$d$ with $d$ prime. Assume that one of the following conditions holds:

a) $n \geqslant 3$, or

b) $\operatorname{char}(k)=0$ and $Y$ is a generic surface in $P^{3}$ with $d \geqslant 5$.

Assume further that $Y$ is embedded as an ample divisor in the projective smooth variety $X$. Then one has one of the following possibilities:

i) $X$ is isomorphic to $P^{n+1}$ and the inclusion $Y \subset X$ is just $H(d) \subset$ $P^{n+1}$.

ii) $X$ is isomorphic to a smooth hypersurface of degree $d$ in $P^{n+2}$ and $Y$ is the intersection of $X$ with a hyperplane.

Proof. In case a) by Lefschetz's theorem we have Pic $(Y)=Z\left[O_{Y}(1)\right]$. Also, since $Y=H(d)$ and $\operatorname{dim}(Y)=n \geqslant 3, H^{i}\left(O_{Y}(s)\right)=0$ for $i=1,2$ and for every $s \in Z$; in particular, $H^{i}\left(O_{X}(-m Y) / O_{X}(-(m+1) Y)\right)=0$ for $i=$ 1,2 and for every $m \geqslant 1$. Thus we may apply Lefschetz's theorem to $(X, Y)$ and get that the map $\alpha: \operatorname{Pic}(X) \rightarrow \operatorname{Pic}(Y)$ is an isomorphism.

In case b) we may apply Noether's theorem (see [8], p. 182) and also deduce that $\operatorname{Pic}(Y)=Z\left[O_{Y}(1)\right]$. By [2] $\alpha$ is injective and Coker $(\alpha)$ is torsion-free. Hence $\alpha$ turns out to be also an isomorphism.

Therefore in both cases there is an invertible $O_{X}$-module $L$ such that $L \otimes O_{Y}=O_{Y}(1)$. Further there is an integer $r>0$ such that $O_{X}(Y) \cong L^{\otimes r}$. Let $\sigma \in \Gamma\left(X, O_{Y}(Y)\right) \cong \Gamma\left(X, L^{\otimes r}\right)$ be a section such that $\operatorname{div}_{X}(\sigma)=Y$. We have

$$
\begin{aligned}
\left(L^{\cdot(n+1)}\right)_{X} & =1 / r \cdot\left(L^{\cdot n} \cdot L^{\otimes r}\right)_{X}=1 / r \cdot\left(L^{\cdot n} \cdot Y\right)_{X}=1 / r \cdot\left(L_{Y}^{n}\right)_{Y} \\
& =1 / r \cdot\left(O_{Y}(1)^{\cdot n}\right)_{Y}=d / r, \quad \text { where } L_{Y}=L \otimes O_{Y} .
\end{aligned}
$$

In particular $r$ divides $d$, and since $d$ is prime one has two possibilities.

1) $r=d$, i.e. $O_{X}(Y)=L^{\otimes d}$.

Then (4) gives $\left(L^{\cdot(n+1)}\right)_{Y}=1$. On the other hand, exactly as in the proof of Theorem 1 one shows that the sequence

$$
0 \longrightarrow \Gamma\left(L^{\otimes(1-d)}\right) \stackrel{\sigma}{\longrightarrow} \Gamma(L) \longrightarrow \Gamma\left(O_{Y}(1)\right) \longrightarrow 0
$$

is exact. Since $d>1$ and $L$ is ample $\Gamma\left(L^{\otimes(1-d)}\right)=0$. Thus $\operatorname{dim} \Gamma(L)=$ $n+2$. Now Theorem 3 applied to $V=X$ leads to case i).

2) $r=1$, i.e. $L \cong O_{X}(Y)$.

Again one deduces the exact sequence (for every $m \in \boldsymbol{Z}$ )

$$
0 \longrightarrow \Gamma\left(L^{\otimes(m-1)}\right) \longrightarrow \Gamma\left(L^{\otimes m}\right) \longrightarrow \Gamma\left(O_{Y}(m)\right) \longrightarrow 0 \text {. }
$$

Denoting by $S$ the graded $k$-algebra $\oplus_{m=0}^{\infty} \Gamma\left(X, L^{\otimes m}\right), \sigma \in S_{1}$ and by (5) 
$S / \sigma S \cong \oplus_{m=0}^{\infty} \Gamma\left(Y, O_{Y}(m)\right)$. Recalling that $Y$ is a hypersurface in $P^{n+1}$, this last algebra is generated by its homogeneous part of degree one. Hence $S$ itself is generated by $S_{1}=\Gamma(L)$, and in particular $L$ is very ample on $X$.

If in (5) we take $m=1$ we get $\operatorname{dim} \Gamma(L)=\operatorname{dim} \Gamma\left(O_{X}\right)+\operatorname{dim} \Gamma\left(O_{Y}(1)\right)$ $=n+3$. Therefore the canonical map $\varphi=\varphi_{L}: X \rightarrow P(\Gamma(L))-P^{n+2}$ is a closed immersion. Since $\varphi^{*}(O(1)) \cong L$ (taking into account that $r=1$ and (4))

$$
\operatorname{deg} \varphi(X)=\left(O(1)^{\cdot(n+1)} \cdot \varphi(X)\right)_{P^{n+2}}=\left(L^{\cdot(n+1)}\right)_{X}=d .
$$

The fact that $\varphi(Y)$ is the intersection of $\varphi(X)$ with a hyperplane of $P^{n+2}$ is now clear. Thus case 2) leads to case ii). Q.E.D.

Corollary. Let $Y$ be a hyperquadric in $P^{n+1}$ with $n \geqslant 3$. Then $Y$ can be an ample divisor on the smooth projective variety $X$ if and only if either $X$ is isomorphic to $P^{n+1}$, or to a smooth hyperquadric in $P^{n+2}$.

Remark. If $k=C$ the above corollary has been previously obtained by Sommese in [13].

\section{§3. Lifting to characteristic zero}

Let $k$ be an algebraically closed field of characteristic $p>0$ and $A$ $=W(k)$ the ring of Witt vectors on $k$, which is a complete discrete valuation ring of characteristic zero, with residue field $k$ and such that $p$ generates its maximal ideal. Let $X$ be a projective smooth variety over $k$. One says that $X$ has a lifting to characteristic zero if there is a projective smooth morphism $f: \mathscr{X} \rightarrow \operatorname{Spec}(A)$ whose closed fibre is isomorphic to $X$. Then the generic fibre $X^{\prime}$ of $f$ is a projective smooth variety over the quotient field $k^{\prime}$ of $A$.

Grothendieck proved in [6], exposé III, théorème 7.3 that a sufficient condition for the existence of a lifting to characteristic zero of $X$ is the following " $H^{2}\left(T_{X}\right)=H^{2}\left(O_{X}\right)=0$ ", where $T_{X}=\left(\Omega_{X / k}^{1}\right)^{\vee}$ is the tangent sheaf of $X$.

Let now $k$ be a field (not necessarily algebraically closed) and $X$ a smooth projective variety of dimension 3 over $k$. Let $L$ be an ample invertible $O_{X}$-module and $\sigma \in \Gamma(X, L)$ a section such that $Y=\operatorname{div}_{X}(\sigma)$ is smooth over $k$. Then we have the following result which follows from [2].

Proposition 1. Assume char $(k)=0$. Then the natural map Pic $(X)$ $\rightarrow \operatorname{Pic}(Y)$ is injective and its cokernel is torsion-free. 
Lemma 1. Let $k$ be an algebraically closed field of characteristic $p>0$ and $X, L, \sigma$, and $Y$ as above. Assume moreover:

i) $X$ has a lifting to characteristic zero.

ii) $H^{i}\left(O_{X}\right)=0$ for $i=1,2$.

iii) $H^{i}\left(O_{Y}\right)=0$ for $i=1,2$.

iv) $H^{1}(L)=0$.

Then the map of restriction $\mathrm{Pic}(X) \rightarrow \operatorname{Pic}(Y)$ is injective with cokernel a torsion-free group.

Proof. Let $f: \mathscr{X} \rightarrow \operatorname{Spec}(A)$ be a lifting to characteristic zero of $X$. First we prove that the natural map of restriction $\operatorname{Pic}(\mathscr{X}) \rightarrow \operatorname{Pic}(X)$ is an isomorphsm. Indeed, let $\hat{X}$ be the formal completion of along $X$. Then by Grothendieck's existence theorem (see [3], chap. III 5.4.1) the natural map Pic $(\mathscr{X}) \rightarrow \operatorname{Pic}(\hat{X})$ is an isomorphism. It will be therefore sufficient to show that the map of restriction $\operatorname{Pic}(\hat{X}) \rightarrow \operatorname{Pic}(X)$ is also an isomorphism. Let $\mathscr{X}_{n}$ be the closed subscheme of $\mathscr{X}$ defined by the sheaf of ideals $p^{n} O_{\mathscr{x}}$. In particular $\mathscr{X}_{1}=X$. An invertible $O_{\hat{x}}$-module is nothing but a sequence $\left(L_{n}\right)_{n \geqslant 1}$, where $L_{n}$ in an invertible $O_{x_{n}}$-module, plus isomorphisms $L_{n+1} \otimes$ $O_{x_{n}} \cong L_{n}$. Then the map Pic $(\hat{X}) \rightarrow \operatorname{Pic}(X)$ is precisely $\left(L_{n}\right)_{n \geqslant 1} \sim L_{1}$. In order to see that this map is an isomorphism it will be sufficient to show that for each $n \geqslant 1$ the map of restriction Pic $\left(\mathscr{X}_{n+1}\right) \rightarrow \operatorname{Pic}\left(\mathscr{X}_{n}\right)$ is an isomorphism. But this follows from the standard exact sequence

$$
0 \longrightarrow p^{n} O_{x} / p^{n+1} O_{x} \cong O_{X} \longrightarrow O_{x_{n+1}}^{*} \longrightarrow O_{x_{n}}^{*} \longrightarrow 1,
$$

which together with hypothesis ii) yields the assertion.

In particular there exists an invertible $O_{x}$-module $\mathscr{L}$ such that $\mathscr{L} \otimes$ $O_{x} \cong L$, and by [3], chap. III 4.7.1 $\mathscr{L}$ is ample. Moreover, from the exact sequence

$$
\Gamma(\mathscr{X}, \mathscr{L}) \longrightarrow \Gamma(X, L) \longrightarrow H^{1}(\mathscr{X}, \mathscr{L}) \stackrel{p}{\longrightarrow} H^{1}(\mathscr{X}, \mathscr{L}) \longrightarrow H^{1}(X, L)=0
$$

and Nakayama's lemma we deduce that the first map is surjective. In particular, $\sigma$ lifts to a section $\tau \in \Gamma(\mathscr{X}, \mathscr{L})$. Set $\mathscr{Y}=\operatorname{div}_{\mathscr{x}}(\tau)$ and $g=$ $f / \mathscr{Y}: \mathscr{Y} \rightarrow \operatorname{Spec}(A)$. Then the closed fibre of $g$ is $Y$, and hence $g$ is a smooth morphism. If $Y^{\prime}$ is the generic fibre of $g$, then $Y^{\prime}=\operatorname{div}_{X^{\prime}}\left(\tau / X^{\prime}\right)$ is a smooth surface in $X^{\prime}$. By Proposition 1 the map $\operatorname{Pic}\left(X^{\prime}\right) \rightarrow \operatorname{Pic}\left(Y^{\prime}\right)$ is injective with cokernel a torsion-free group. In order to complete the proof of Lemma 1 it will be therefore sufficient to show that there are isomorphisms 
$\operatorname{Pic}\left(X^{\prime}\right) \stackrel{\sim}{\longrightarrow} \operatorname{Pic}(X)$ and $\operatorname{Pic}\left(Y^{\prime}\right) \stackrel{\sim}{\longrightarrow} \operatorname{Pic}(Y)$

making commutative the following diagram

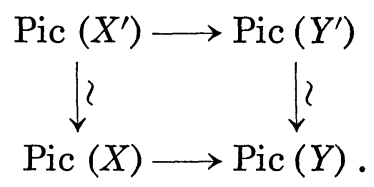

This fact is well known. For example we have firstly the isomorphism Pic $\left(Y^{\prime}\right) \stackrel{\sim}{\longrightarrow}$ Pic $(\mathscr{Y})$ defined by $\left[M 1 \sim\left[M^{\prime}\right]\right.$, where $M^{\prime}$ is an invertible $O_{g}$-module such that $M^{\prime} / Y^{\prime} \cong M$. Such a $M^{\prime}$ always exists because $\mathscr{Y}$ is a regular scheme and $Y^{\prime}$ is an open subset in $\mathscr{Y}$. This definition is correct since the complement of $Y^{\prime}$ is $Y$ and $Y$ is defined as a closed subscheme of $\mathscr{Y}$ by the ideal $p O_{y}$, which is isomorphic as an $O_{y}$-module to $O_{y}$. Secondly, by the first part of the proof the natural map Pic (Y) $\rightarrow \operatorname{Pic}(Y)$ is an isomorphism.

Q.E.D.

Lemma 2. Assume that $Y=P^{2}$ (resp. $Y=P^{1} \times P^{1}$ ) is contained in the smooth projective variety $X$ as an ample divisor, where $k$ is an algebraically closed field of arbitrary characteristic. Then the map of restriction $\operatorname{Pic}(X) \rightarrow \operatorname{Pic}(Y)$ is an isomorphism (resp. is injective and its cokernel is a torsion-free group).

Proof. If char $(k)=0$ this follows directly from Proposition 1 taking into account (if $Y=P^{2}$ ) that $\operatorname{Pic}\left(P^{2}\right) \cong Z$. Assume therefore char $(k)>0$. Then the conclusion will follow from Lemma 1 if we show that conditions i)-iv) are satisfied by $\left(X, L=O_{X}(Y), \sigma\right.$, $\left.\operatorname{div}_{X}(\sigma)=Y\right)$. The verification of conditions ii), iii) and iv) is not difficult (using the explicit computation of the cohomology of $P^{2}$ and $P^{1} \times P^{1}$ and the cohomological characterization of ampleness) and is left to the reader.

In order to verify condition i) it will be sufficient (using [6], exposé III, théorème 7.3) to show that $H^{2}\left(T_{X}\right)=0$ (the condition $H^{2}\left(O_{X}\right)=0$ being contained in ii)). Consider the exact sequence $(m \in Z)$

$H^{1}\left(T_{X} \otimes O_{X}(m Y) \otimes O_{Y}\right) \longrightarrow H^{2}\left(T_{X} \otimes O_{X}((m-1) Y)\right) \longrightarrow H^{2}\left(T_{X} \otimes O_{X}(m Y)\right)$.

Since $Y$ is ample on $X, H^{2}\left(T_{X} \otimes O_{X}(m Y)\right)=0$ for $m \gg 0$. Therefore in order to prove that $H^{2}\left(T_{X}\right)=0$ it will be sufficient (via descending induction on $m$ ) to see that

$$
H^{1}\left(T_{X} \otimes O_{X}(m Y) \otimes O_{Y}\right)=0 \quad \text { for every } m \geqslant 1
$$


Consider the exact sequence

$$
\begin{aligned}
H^{1}\left(T_{Y} \otimes O_{X}(m Y)\right) & \longrightarrow H^{1}\left(T_{X} \otimes O_{X}(m Y) \otimes O_{Y}\right) \\
& \longrightarrow H^{1}\left(O_{X}((m+1) Y) \otimes O_{Y}\right)
\end{aligned}
$$

(induced by $0 \rightarrow T_{Y} \rightarrow T_{X} \otimes O_{Y} \rightarrow O_{X}(Y) \otimes O_{Y} \rightarrow 0$ ).

If $Y=P^{2}$ then $O_{X}(Y) \otimes O_{Y}=O(s)$ with $s>0$ (since $Y$ is ample on $X)$. Then $H^{1}\left(O_{X}((m+1) Y) \otimes O_{Y}\right)=H^{1}\left(P^{2}, O((m+1) s)\right)=0$ for every $m \in$ $Z$. On the other hand, the standard exact sequence on $Y=P^{2}$

$$
0 \longrightarrow O_{Y} \longrightarrow O(1)^{\oplus 3} \longrightarrow T_{Y} \longrightarrow 0
$$

yields the exact sequence of cohomology

$$
0=H^{1}\left(O(m s+1)^{\oplus 3}\right) \longrightarrow H^{1}\left(T_{Y} \otimes O(m s)\right) \longrightarrow H^{2}(O(m s))=0 .
$$

Therefore $H^{1}\left(T_{Y} \otimes O_{X}(m Y)\right)=H^{1}\left(T_{Y} \otimes O(m s)\right)=0$. Now the exact sequence (7) proves (6) if $Y=P^{2}$.

If $Y=P^{1} \times P^{1}$ then $O_{X}(Y) \otimes O_{Y}=O(a, b)$ with $a>0$ and $b>0$. Then $H^{1}\left(O_{X}((m+1) Y) \otimes O_{Y}\right)=H^{1}\left(P^{1} \times P^{1}, O((m+1) a,(m+1) b)\right)=0$ for every $m \geqslant 0$.

On the other hand, $T_{Y} \cong O(2,0) \oplus O(0,2)$, and therefore

$$
H^{1}\left(T_{Y} \otimes O_{Y}(m Y)\right)=H^{1}(O(m a+2, m b)) \oplus H^{1}(O(m a, m b+2))=0 .
$$

Again the exact sequence (7) proves (6) if $Y=P^{1} \times P^{1}$.

Q.E.D.

Proposition 2. Assume that $Y=P^{2}$ is embedded as an ample divisor in the smooth projective variety $X$. Then $X$ is isomorphic to $P^{3}$ and $Y$ is contained in $X$ as a hyperplane.

Proof. If char $(k)=0$ this result is contained in Theorem 1. Thus we may assume char $(k)>0$. Since $\operatorname{Pic}\left(P^{2}\right)=Z$ we may apply Lemma 2 and deduce that the map $\operatorname{Pic}(X) \rightarrow \operatorname{Pic}(Y)$ is an isomorphism. Now the argument is contained in the proof of Theorem 1.

Q.E.D.

TheORem 5. Assume that $Y=P^{1} \times P^{1}$ is embedded in the smooth projective variety $X$ as an ample divisor. Then we have one of the following possibilities:

i) $X \cong P^{3}$ and $Y$ is a quadric in $X$.

ii) $X$ is isomorphic to a hyperquadric in $P^{4}$ and $Y$ is a hyperplane section.

iii) There are $a>0, b>0, c>0$ and $s>0$ positive integers such that $a+b+c=2 s$ and the exact sequence of $O_{P 1}$-modules 


$$
0 \longrightarrow O_{P 1} \longrightarrow O(a) \oplus O(b) \oplus O(c)=E \stackrel{\varphi}{\longrightarrow} O(s) \oplus O(s)=F \longrightarrow 0
$$

such that $X$ is isomorphic to $P(E)$ and $Y \cong P(F)$ is embedded in $X$ via the surjection $\varphi$.

Proof. From Lemma 2 we deduce that the map

$$
\operatorname{Pic}(X) \stackrel{\alpha}{\longrightarrow} \operatorname{Pic}(Y) \cong Z \times Z
$$

is injective and its cokernel is torsion-free. Thus we have two possibilities:

a) $\operatorname{Pic}(X) \cong Z$. Let $L$ be an invertible $O_{X}$-module which is ample and generates Pic $(X)$. Then $L \otimes O_{Y} \cong O(s, t)$ with $s>0$ and $t>0$. Since Coker $(\alpha)$ is torsion-free $s$ and $t$ are relatively prime integers. Writing $O_{X}(Y) \cong L^{\otimes r}$ and $\omega_{X} \cong L^{\otimes d}$, we get easily from the adjunction formula that $s(d+r)=t(d+r)=-2$, and thus $s=t=1$.

Let $\sigma \in \Gamma\left(X, O_{X}(Y)\right) \cong \Gamma\left(X, L^{\otimes r}\right)$ be such that $\operatorname{div}_{X}(\sigma)=Y$. The exact sequence

$$
0 \longrightarrow L^{\otimes(m-r)} \stackrel{\sigma}{\longrightarrow} L^{\otimes m} \longrightarrow O(m, m) \longrightarrow 0
$$

yields the exact sequence $(m \in Z)$

$$
0 \longrightarrow \Gamma\left(L^{\otimes(m-r)}\right) \stackrel{\sigma}{\longrightarrow} \Gamma\left(L^{\otimes m}\right) \longrightarrow \Gamma(O(m, m)) \longrightarrow H\left(L^{\otimes(m-r)}\right)=0 .
$$

Put $S=\oplus_{m=0}^{\infty} \Gamma\left(L^{\otimes m}\right)$; then $S / \sigma S \cong \bigoplus_{m=0}^{\infty} \Gamma(O(m, m))$ is a graded $k$ algebra generated by its part of degree one. On the other hand

$$
\left(L^{\cdot 3}\right)_{X}=1 / r \cdot\left(L^{\cdot 2} \cdot Y\right)_{X}=1 / r \cdot(O(1,1) \cdot O(1,1))_{Y}=2 / r .
$$

Therefore $r=2$ or $r=1$.

$\left.a_{1}\right)$ Case $r=2$. If in (8) we take $m=1$ we get $\operatorname{dim} \Gamma(L)=4$. Since $\left(L^{\cdot 3}\right)_{X}=1$ Theorem 3 implies $X=P^{3}$ and we get case i).

$a_{2}$ ) Case $r=1$. Then $\operatorname{deg}(\sigma)=1$ and since $S / \sigma S$ is generated by its homogeneous part of degree one, the same is true for $S$. In particular $L$ is very ample. Again take $m=1$ in (8) and get $\operatorname{dim} \Gamma(L)=5$. Thus $\varphi_{L}: X \rightarrow P(\Gamma(L)) \cong P^{4}$ and since $\operatorname{deg} \varphi_{L}(X)=2$ we get case ii).

b) $\operatorname{Pic}(X) \cong Z \times Z$. Then the map $\operatorname{Pic}(X) \stackrel{\alpha}{\longrightarrow} \operatorname{Pic}(Y)$ is an isomorphism. Therefore there are two invertible $O_{X}$-modules $L_{1}$ and $L_{2}$ such that $L_{1} \otimes O_{Y} \cong O(1,0)$ and $L_{2} \otimes O_{Y}=O(0,1)$. If $O_{X}(Y) \otimes O_{Y} \cong O\left(s_{1}, s_{2}\right)$ with $s_{1}>0$ and $s_{2}>0$ ( $Y$ is ample on $X$ ), then since the map $\alpha$ is injective, $O_{X}(Y) \cong L_{1}^{\otimes s_{1}} \otimes L_{2}^{\otimes s_{2}}$. Let $\sigma \in \Gamma\left(O_{X}(Y)\right) \cong \Gamma\left(L_{1}^{\otimes s_{1}} \otimes L_{2}^{\otimes s_{2}}\right)$ be a section such 
that $\operatorname{div}_{X}(\sigma)=Y$. Then the exact sequence

$$
0 \longrightarrow O_{X}((m-1) Y) \stackrel{\sigma}{\longrightarrow} O_{X}(m Y) \longrightarrow O\left(m s_{1}, m s_{2}\right) \longrightarrow 0
$$

yields the exact sequence (exactly as in the proof of Theorem 1)

$$
0 \longrightarrow \Gamma\left(O_{X}((m-1) Y)\right) \stackrel{\sigma}{\longrightarrow} \Gamma\left(O_{X}(m Y)\right) \longrightarrow \Gamma\left(O\left(m s_{1}, m s_{2}\right)\right) \longrightarrow 0 \text {. }
$$

Put $S=\bigoplus_{m=0}^{\infty} \Gamma\left(O_{X}(m Y)\right)$; then $\sigma \in S_{1}$ and $S / \sigma S \cong \bigoplus_{m=0}^{\infty} \Gamma\left(Y, O\left(m s_{1}, m s_{2}\right)\right)$ is generated by its homogeneous part of degree one. Therefore $S$ itself is generated by $S_{1}$ and hence $Y$ is very ample on $X$. If in (9) we take $m=1$ we get

$$
\operatorname{dim}|Y|=\left(s_{1}+1\right)\left(s_{2}+1\right)
$$

If $s_{1}=s_{2}=1$ then $|Y|=P^{4}$ and $X$ would be a smooth hypersurface in $P^{4}$. But then Lefschetz's theorem yields $\operatorname{Pic}(X) \cong Z$, a contradiction. Thus at least one $s_{1}$ or $s_{2}$ is $>1$.

Suppose $s_{1}>1$. Then the exact sequence

$$
0 \longrightarrow L_{1}^{\otimes\left(1-s_{1}\right)} \otimes L_{2}^{\left(-s_{2}\right)} \stackrel{\sigma}{\longrightarrow} L_{1} \longrightarrow O(1,0) \longrightarrow 0
$$

yields the exact sequence

$$
\begin{aligned}
0 \longrightarrow \Gamma\left(L_{1}^{\otimes\left(1-s_{1}\right)} \otimes L_{2}^{\otimes\left(-s_{2}\right)}\right) \longrightarrow & \Gamma\left(L_{1}\right) \longrightarrow \Gamma(O(1,0)) \\
& \longrightarrow H^{1}\left(L_{1}^{\otimes\left(1-s_{1}\right)} \otimes L_{2}^{\otimes\left(-s_{2}\right)}\right) .
\end{aligned}
$$

Since $1-s_{1}<0$ and $-s_{2}<0$ we have $H^{i}\left(L_{1}^{\otimes\left(1-s_{1}\right)} \otimes L_{2}^{\otimes\left(-s_{2}\right)}\right)=0$ for $i \leqslant 1$. Indeed, $H^{i}\left(F \otimes O_{X}(m Y)\right)=0$ for $i \leqslant 1$ and $m \ll 0$ (with $F=L_{1}$ ), and from the exact sequence

$$
0 \longrightarrow F \otimes O_{X}((m-1) Y) \longrightarrow F \otimes O_{X}(m Y) \longrightarrow O\left(m s_{1}+1, m s_{2}\right) \longrightarrow 0
$$

we deduce for every $m<0$ and $i \leqslant 1$ :

$$
H^{i}\left(F \otimes O_{X}((m-1) Y)\right) \longrightarrow H^{i}\left(F \otimes O_{X}(m Y)\right) \longrightarrow H^{i}\left(O\left(m s_{1}+1, m s_{2}\right)\right) .
$$

By Künneth's formulae we get $H^{i}\left(O\left(m s_{1}+1, m s_{2}\right)\right)=0$ for $i \leqslant 1$ and $m<0$, and the affirmation results by induction on $m$.

Now recalling (11) we get that the map of restriction $\Gamma\left(L_{1}\right) \rightarrow \Gamma(O(1,0))$ is an isomorphism if $s_{1}>1$. In particular, for every $\Delta, \Delta^{\prime} \in\left|L_{1}\right|\left(\Delta \neq \Delta^{\prime}\right)$ we have $\Delta \cap \Delta^{\prime} \cap Y=\phi$. Since $Y$ is ample on $X, \Delta \cap \Delta^{\prime}$ is at most a finite set of closed points. Since $X$ is smooth we cannot have $\Delta \cap \Delta^{\prime} \neq \phi$ because otherwise 


$$
3=\operatorname{codim}_{X}\left(\Delta \cap \Delta^{\prime}\right) \leqslant \operatorname{codim}_{X}(\Delta)+\operatorname{codim}_{X}\left(\Delta^{\prime}\right)=1+1=2 .
$$

Therefore $\Delta \cap \Delta^{\prime}=\phi$. Thus the linear system $\left|L_{1}\right|$ has no base points and hence the corresponding map $p=\varphi_{L_{1}}: X \rightarrow\left|L_{1}\right|=P^{1}$ (such that $p^{*} O_{P 1}(1)$ $\left.\cong L_{1}\right)$ is a morphism. Moreover, for every invertible $O_{X}$-module $L,\left(L_{1}^{\cdot 2} \cdot L\right)$ $=0$.

Now look at the equalities

$$
1=(O(1,0) \cdot O(0,1))_{Y}=\left(L_{1} \cdot L_{2} \cdot Y\right)_{X}=s_{1}\left(L_{1}^{\cdot 2} \cdot L_{2}\right)+s_{2}\left(L_{1} \cdot L_{2}^{2}\right) .
$$

One deduces $s_{2}\left(L_{1} \cdot L_{2}^{\cdot 2}\right)=1$, i.e. $s_{2}=1$ and $\left(L_{1} \cdot L_{2}^{\cdot 2}\right)=1$. Set $s_{1}=s$.

Let $\Delta \in\left|L_{1}\right|$ be arbitrary. Then $\left(O_{X}(Y)^{\cdot 2} \cdot \Delta\right)=s^{2}\left(L_{1}^{\cdot 3}\right)+2 s\left(L_{1}^{\cdot 2} \cdot L_{2}\right)+$ $\left(L_{1} \cdot L_{2}^{2}\right)=\left(L_{1} \cdot L_{2}^{2}\right)=1$. Therefore, denoting by $M=O_{X}(Y) \otimes O_{\Delta}$, we get $\left(M^{2}\right)_{\Delta}=1, M$ is ample on $\Delta$ and $\Delta$ is a Cohen-Macaulay scheme of pure dimension 2. Moreover, for every $i=0,1, \cdots, s-1$ one has the exact sequence (since $L_{1} \otimes O_{\Delta} \cong O_{\Delta}$ )

$$
0 \longrightarrow L_{1}^{\otimes(s-i-1)} \otimes L_{2} \longrightarrow L_{1}^{\otimes(s-i)} \otimes L_{2} \longrightarrow M \longrightarrow 0
$$

and hence

$$
0 \longrightarrow \Gamma\left(L_{1}^{\otimes(s-i-1)} \otimes L_{2}\right) \longrightarrow \Gamma\left(L_{1}^{\otimes(s-i)} \otimes L_{2}\right) \longrightarrow \Gamma(M) .
$$

Claim. $\operatorname{Dim} \Gamma(M) \geqslant 3$.

Indeed, assuming the contrary we get

$$
2 \geqslant \operatorname{dim} \Gamma\left(L_{1}^{\otimes(s-i)} \otimes L_{2}\right)-\operatorname{dim} \Gamma\left(L_{1}^{\otimes(s-i-1)} \otimes L_{2}\right), \quad i=0,1, \cdots, s-1,
$$

and therefore taking the sum:

$$
2 s \geqslant \operatorname{dim} \Gamma\left(O_{X}(Y)\right)=\operatorname{dim} \Gamma\left(L_{2}\right) .
$$

But the exact sequence

$$
0 \longrightarrow L_{1}^{\otimes(-s)} \stackrel{\sigma}{\longrightarrow} L_{2} \longrightarrow O(0,1) \longrightarrow 0
$$

yields

$$
0=\Gamma\left(L_{1}^{\otimes(-s)}\right) \longrightarrow \Gamma\left(L_{2}\right) \longrightarrow \Gamma(O(0,1))
$$

and thus $\operatorname{dim} \Gamma\left(L_{2}\right) \leqslant 2$. Therefore (12) becomes $\operatorname{dim} \Gamma\left(O_{X}(Y)\right) \leqslant 2(s+1)$, or else $\operatorname{dim}|Y| \leqslant 2 s+1$, which contradicts (10). The claim is proved.

By Theorem 3 we deduce then that $\Delta \cong P^{2}$ and $O_{\Delta}(1) \cong L_{2} \otimes O_{\Delta}$. Now Hironaka has shown that in these circumstances $p$ is the projection of the projective bundle $P(E)$ associated to a locally free $O_{P^{1}}$-module $E$ of 
rank 3 (see [9], Theorem (1.8)). Moreover $O_{X}(Y) \otimes O_{\Delta} \cong L_{1}^{\otimes s} \otimes L_{2} \otimes O_{\Delta} \cong$ $L_{2} \otimes O_{4} \cong O_{4}(1)$, and therefore we can take $E=p_{*} O_{X}(Y)$. Then $O_{P(E)}(1)=$ $O_{X}(Y)$ and the exact sequence

$$
0 \longrightarrow O_{X} \longrightarrow O_{X}(Y) \longrightarrow O(s, 1) \longrightarrow 0
$$

yields

$$
\begin{aligned}
0 \longrightarrow & p_{*} O_{X} \cong O_{P^{1}} \longrightarrow p_{*} O_{X}(Y)=E \\
& \longrightarrow p_{1 *} O(s, 1) \cong O(s) \oplus O(s) \longrightarrow R^{1} p_{*} O_{X}=0,
\end{aligned}
$$

where $p_{1}: P^{1} \times P^{1} \rightarrow P^{1}$ is the first projection. In other words we get the exact sequence of locally free $O_{P 1}$-modules

$$
0 \longrightarrow O_{P 1} \longrightarrow E \longrightarrow O(s) \oplus O(s) \longrightarrow 0 \text {. }
$$

In particular deg $(E)=2 s$. By a theorem of Grothendieck (see [4] for $k$ $=C$, but the same result holds in arbitrary characteristic) there are three integers $a, b, c$ (uniquely determined up to a permutation) such that $E \cong$ $O(a) \oplus O(b) \oplus O(c)$. Finally, since $O_{X}(Y)$ is ample on $X, E$ is ample on $P^{1}$, and therefore $a>0, b>0$ and $c>0$. In other words we get situation iii).

Q.E.D.

Remarks. 1) The case iii) of Theorem 5 really occurs. Indeed, we shall construct an exact sequence as in case iii) with $c=s$, i.e. with $a+$ $b=s(a>0, b>0$ and $c>0)$. It will be sufficient to construct a surjection of the form $\varphi^{\prime}: O(a) \oplus O(b) \rightarrow O(a+b)=O(s)$, because one can take $\varphi=\varphi^{\prime} \oplus \mathrm{id}_{O(s)}$ (and then taking the degrees one sees that $\operatorname{Ker}(\varphi) \cong O_{P^{1}}$ ). Let $x_{0}$ and $x_{1}$ homogeneous coordinates on $P^{1}$ and define $\varphi^{\prime}(p, q)=x_{0}^{b} p+$ $x_{1}^{a} q$. We claim that $\Gamma\left(\varphi^{\prime}\right): \Gamma(O(a)) \oplus \Gamma(O(b)) \rightarrow \Gamma(O(a+b))$ is surjective. For, if $u \in \Gamma(O(a+b))=k\left[x_{0}, x_{1}\right]_{a+b}$ is of the form $u=\sum_{i=0}^{a+b} a_{i} x_{0}^{i} x_{1}^{a+b-i}$, then $u=x_{0}^{b} p+x_{1}^{a} q$, where $p=\sum_{i=0}^{a-1} a_{i} x_{0}^{i} x_{1}^{a-i} \in \Gamma(O(a))$ and $q=\sum_{i=a}^{a+b} a_{i} x_{0}^{i-a} x^{a+b-i}$ $\in \Gamma(O(b))$. Now since $\Gamma\left(\varphi^{\prime}\right)$ is surjective and $O(a+b)$ is generated by its global sections, $\varphi^{\prime}$ is also surjective (and thus $\varphi$ is surjective).

2) Note that the theorem asserting that $P^{n}$ is the unique smooth projective variety containing $P^{n-1}(n \geqslant 3)$ as an ample divisor was known for $n \geqslant 4$ and char $(k)$ arbitrary, and for $n=3$ and char $(k) \neq 3$ (see [12]).

Added in proof. 1. Further results in connection with the problem of ample divisors can be found in author's paper "On ample divisors: II", Proceedings of the Week of Algebraic Geometry, Bucharest 1980, pp. 12-32, 
Teubner-Texte zur Mathematik, Band 40, Leipzig 1981. We would also like to mention the paper of T. Fujita "On the hyperplane section principle of Lefschetz", J. Math. Soc. Japan 32 (1980) 153-169.

2. We are indebted to Professor A. Franchetta for informing us about a classical result of $\mathrm{G}$. Scorza, which, although stated in a different form, turns out to be equivalent to our theorem 1 above.

3. Theorem 4 above can be also deduced from Mori's work [12].

\section{REFERENCES}

[1] Altman, A. and Kleiman, S., Introduction to Grothendieck duality theory, Springer Lect. Notes Math., 146 (1970).

[2] Bădescu, L., A remark on the Grothendieck-Lefschetz theorem about the Picard group, Nagoya Math. J., 71 (1978), 169-179.

[ 3 ] Dieudonné, J. and Grothendieck, A., Eléments de Géométrie Algébrique, Publ. Math. IHES, 11 (1961).

[4] Grothendieck, A., Sur la classification des fibrés holomorphes sur la sphére de Riemann, Amer. J. Math., 79 (1957), 121-138.

[ 5 ] — Local cohomology, Springer Lect. Notes Math., 41 (1967).

[ 6 ] - Revetements étales et groupe fondamental, Springer Lect. Notes Math., 221 (1971).

[ 7 ] - Cohomologie locale des faisceaux cohérents et théorèmes de Lefschetz locaux et globaux, North-Holland, Amsterdam (1968).

[ 8 ] Hartshorne, R., Ample subvarieties of algebraic varieties, Springer Lect. Notes Math., 156 (1970).

[9 ] Hironaka, H., Smoothing of algebraic cycles of small dimensions, Amer. J. Math., 90 (1968), 1-54.

[10] Kleiman, S., Toward a numerical theory of ampleness, Annals Math., 84 (1966), 293-344.

[11] Kobayashi, S. and Ochiai, T., Characterizations of complex projective spaces and hyperquadrics, J. Math. Kyoto Univ., 13-1 (1973), 31-47.

[12] Mori, S., On a generalization of complete intersections, J. Math. Kyoto Univ., 15-3 (1975), 619-646.

[13] Sommese, A. J., On manifolds that cannot be ample divisors, Math. Ann., 221 (1976), 55-72.

I.N.C.R.E.S.T. Bucharest

Department of Mathematics

$B$-dul Pacii 220

77538 Bucharest, Romania 